Submitted to ApJ

\title{
Updated Estimates of Global Cosmological Parameters Determined Using Classical Double Radio Galaxies
}

\author{
Erick J. Guerra ${ }^{1}$, Ruth A. Daly ${ }^{2,3}$, and Lin Wan \\ Department of Physics, Princeton University, Princeton, NJ 08544
}

\begin{abstract}
A sample of 20 powerful extended radio galaxies with redshifts between zero and two were used to determine constraints on global cosmological parameters. Data for six radio sources were obtained from the VLA archive, analyzed, and combined with the sample of 14 radio galaxies used previously by Guerra \& Daly to determine cosmological parameters. The new results are consistent with our previous results, and indicate that the current value of the mean mass density of the universe is significantly less than the critical value. A universe with $\Omega_{m}$ in matter of unity is ruled out at $99.0 \%$ confidence, and the best fitting values of $\Omega_{m}$ in matter are $0.10_{-0.10}^{+0.25}$ and $-0.25_{-0.25}^{+0.35}$ assuming zero space curvature and zero cosmological constant, respectively.

The radio properties of each source are also used to determine the density of the gas in the vicinity of the source, and the beam power of the source. The six new radio sources have physical characteristics similar to those found for the original 14 sources. The density of the gas around these radio sources is typical of gas in present day clusters of galaxies. The beam powers are typically about $10^{45} \mathrm{erg} \mathrm{s}^{-1}$.
\end{abstract}

Subject headings: cosmology: observations — galaxies: active, evolution, jets radio continuum: galaxies

\footnotetext{
${ }^{1}$ Present address: Department of Chemistry and Physics, Rowan University, Glassboro, NJ 08028; eddie@scherzo.rowan.edu

${ }^{2}$ Present address: Department of Physics, Bucknell University, Lewisburg, PA 17837; daly@bucknell.edu

${ }^{3}$ National Young Investigator
} 


\section{Introduction}

The future and ultimate fate of the universe can be predicted given a knowledge of the recent expansion history of the universe (assuming the universe is homogeneous and isotropic on scales greater than the current horizon size). This recent expansion history can be probed by studying the coordinate distance to sources at redshifts of one or two; the coordinate distance is equivalent to the luminosity distance or angular size distance multiplied by factors of $(1+z)$. The advantage of determining cosmological parameters using the coordinate distance (luminosity distance or angular size distance) is that this distance depends on global, or average, cosmological parameters. It is independent of the way the matter is distributed spatially, of the power spectrum of density fluctuations, of whether the matter is biased relative to the light, and of the form or nature of the dark matter (assuming that the universe is homogeneous and isotropic on large scales).

It was shown in 1994 that powerful double-lobed radio galaxies provide a modified standard yardstick that can be used to determine global cosmological parameters (Daly 1994, 1995), much like supernovae can be used as modified standard candles. The method was applied and discussed in detail by Guerra \& Daly (1996, 1998), Guerra (1997), and Daly, Guerra, \& Wan (1998) who found that the data strongly favor a low density universe; a universe with $\Omega_{m}=1$ was ruled out at $97.5 \%$ confidence.

It was shown by Daly $(1994,1995)$ that the radio properties of these sources could be used not only to study global cosmological parameters, but also to determine the ambient

gas density, beam power, Mach number of lobe advance, and ambient gas temperature of the sources and their environments. The characteristics of the sources and their environments are presented and discussed in a series of papers (Wellman \& Daly 1996a,b; Wan, Daly, \& Wellman 1996; Daly 1996; Wellman, Daly, \& Wan 1997a,b; Wan \& Daly 1998a,b; Wan, Daly, \& Guerra 1998).

Radio maps of six powerful double-lobed radio galaxies were extracted from the Very Large Array (VLA) archives at the National Radio Astronomy Observatory (NRAO), and analyzed in detail. New results on global cosmological parameters, ambient gas densities, and beam powers are presented here.

The expanded sample is described in $\S 2$. The new results are presented in $\S 3$. The implications of the results are discussed in $\S 4$. 


\section{Expanded Sample}

Each powerful extended radio galaxy (also known as a "classical double") has a characteristic size, $D_{*}$, that predicts the lobe-lobe separation at the end of its lifetime (Daly 1994, 1995; Guerra \& Daly 1998). The parameters needed to compute the characteristic size are the lobe propagation velocity, $v_{L}$, the lobe width, $a_{L}$, and the lobe magnetic field strength, $B_{L}$. These three parameters can be determined using radio maps with arc-second resolution at multiple frequencies, such as those produced with the VLA or MERLIN (Multi-Element Radio Linked Interferometer Network). Multiple-frequency data are needed to use the theory of spectral aging to estimate the lobe propagation velocity (e.g., Myers \& Spangler 1985). In addition, these maps must have the necessary angular resolution and dynamic range to image sufficient portions of the radio bridges.

Two published data sets, Leahy, Muxlow, \& Stephens (1989) and Liu, Pooley, \& Riley (1992), have radio maps of powerful extended radio galaxies at multiple frequencies which are sufficient to compute all three parameters used in determining $D_{*}$. These data were used to compute $D_{*}$ for 14 radio galaxies (Guerra \& Daly 1996, 1998; Guerra 1997; Daly, Guerra, \& Wan 1998). Current efforts to expand the data set are underway, and include searches through the VLA archive. The VLA archive search has already yielded the desired data for six radio galaxies, and new results including these six sources are presented here.

Data were selected from observations of powerful extended radio galaxies from the $3 \mathrm{CR}$ sample (Bennett 1962) on the basis of the observation frequency and array configuration used. An observation in the VLA archive was a candidate if the lobe-lobe angular size of the source was 10 to 40 times the implied beam size, and four hours separated the first and last scans. These observations should resolve the source sufficiently and have enough $u v$-coverage to image the bridges. From candidate observations at both $\mathrm{L}$ and $\mathrm{C}$ band, we have successfully imaged data from six sources. The VLA archive data sets used here are listed in Table 1.

Radio imaging was performed using the NRAO $\mathcal{A I P S}$ software package. The uv data needed minimal editing, and initial calibration was performed in the standard manner using $\mathcal{A I P S}$. As diagnostics, initial images were made using the $\mathcal{A} \mathcal{I P S}$ task IMAGR both without

and with the CLEAN deconvolution algorithm. The final radio maps were produced with the SCMAP task, which performs self-calibration along with the IMAGR and CLEAN tasks.

Parameters used in $\mathcal{A I P S}$ for imaging, deconvolution, and calibration are chosen to produce radio maps for a given source that can easily be used for spectral aging analysis. This is particularly important where observations at different frequencies are produced by different observers. Although some of these radio maps exist in published form, different 
choices of parameters (such as the restoring beam) are often used to produce these radio maps. Thus, reducing the raw $u v$ data insures that the data analysis can be performed consistently between radio maps.

The computation of $a_{L}, B_{L}$, and $v_{L}$ were performed here in the same manner as Wellman, Daly, \& Wan (1997a,b). The deconvolved lobe width, $a_{L}$, is measured $10 h^{-1}$ kpc from the hot spot toward the host galaxy. The lobe magnetic field strength, $B_{L}$, is computed from the deconvolved surface brightness and bridge width measured $10 h^{-1} \mathrm{kpc}$ from the hot spot toward the host galaxy. The lobe propagation velocity, $v_{L}$, is computed on the basis of spectral aging along the imaged portions of the bridge, and the magnetic field used in spectral aging is computed using values measured $10 h^{-1} \mathrm{kpc}$ and $25 h^{-1} \mathrm{kpc}$ from the hotspot. In this paper, all parameters are computed with $b=0.25$ which means magnetic fields are computed to be 0.25 times the minimum energy values, and without an $\alpha-z$ correction which refers to a correction related to the observed correlation between spectral index and redshift (see Wellman 1997; Wellman, Daly, Wan 1997a,b; Guerra 1998; Guerra \& Daly 1998 for details). It was found by Guerra \& Daly (1998) that constraints on cosmological parameters did not depend on these choices, and very similar results are obtained independent of the value of $b$ and of whether an $\alpha-z$ correction is applied.

These three parameters are computed for each bridge, with the exception of one bridge in 3C 324 which was not imaged along its length sufficiently. The characteristic core-lobe size, $r_{*}$, was computed for each bridge using using the equation (see Guerra \& Daly 1998):

$$
r_{*} \propto\left(\frac{1}{B_{L} a_{L}}\right)^{2 \beta / 3} v_{L}^{1-\beta / 3} .
$$

The characteristic (lobe-lobe) size, $D_{*}$, is taken to be the sum of both $r_{*}$ (or in the case of $3 \mathrm{C} 324, D_{*}=2 r_{*}$ ), and is normalized so that $D_{*}$ of Cygnus A (3C 405), a very low redshift source in our sample, is equal to the average size of the full population of powerful classical double radio galaxies at very low redshift. Constraints on cosmological parameters determined using this method are independent of the normalization of $D_{*}$. As discussed in $\$ 3.1$, the best fit value of the one model parameter $\beta$ is determined simultaneously with the best fit values for the two cosmological parameters that enter, $\Omega_{m}$ and $\Omega_{\Lambda}$, the normalized values of the current values of the mean mass density and the cosmological constant (see Guerra \& Daly 1998; Daly, Guerra, \& Wan 1998).

Table 2 presents the six new $D_{*}$ values in the last column, assuming the best fit value of $\beta=1.75$ (see $\$ 3.1$ ). Source name and redshift are listed in the first two columns. The third column lists the redshift bin corresponding to the assignments in Guerra \& Daly (1998) and Table 3 below. The lobe-lobe angular size of the source is listed in the fourth column, and the fifth and sixth columns list the core-lobe characteristic sizes, $r_{*}$. 


\section{Updated Results}

\subsection{Constraints on Cosmological Parameters}

The subsample of sources with estimates of the characteristic size, $D_{*}$, has been increased from 14 to 20, as discussed above in $\$ 2$. Only sources with physical sizes, defined as the projected separation between the radio hot spots, greater than $20 h^{-1} \mathrm{kpc}$ can be used to determine a characteristic size. This is because smaller sources are typically not sufficiently resolved that the radio data are useful, and the radio lobes of smaller sources are interacting with the interstellar medium of the host galaxy rather than the intergalactic/intracluster medium. It was decided that this same criterion should be applied to the larger comparison sample of powerful $3 \mathrm{CR}$ radio galaxies. Thus, the sample of radio galaxies used to determine the redshift evolution of the physical size has been reduced from 82 to 70; twelve radio galaxies were cut because the physical separation between their lobes was less than $20 h^{-1} \mathrm{kpc}$. This has a rather small impact on the actual means and standard deviations of the parent population, as can be seen by comparing Table 3 of this paper with Table 1 of Guerra \& Daly (1998). The average lobe-lobe separations as a function of redshift are listed in Table 3 for three example choices of cosmological parameters (matter-dominated, curvature-dominated, and spatially flat with non-zero cosmological constant).

To solve simultaneously for the model parameter $\beta$ and the cosmological parameters $\Omega_{m}$ and $\Omega_{\Lambda}$, the ratio of $D_{*}$ for each source to $\langle D\rangle$, the average lobe-lobe size of the parent population in the corresponding redshift bin, is fit to a constant, independent of redshift. The value of the constant is a free parameter, so the normalization of Cygnus A does not affect the fits in any way. Figure 1 illustrates the cosmological dependence of $\langle D\rangle / D_{*}$ on the coordinate distance $\left(a_{o} r\right)$. For $\beta=1.75,\langle D\rangle / D_{*}$ is proportional to $\left(a_{o} r\right)^{1.6}$; thus $\left(\langle D\rangle / D_{*}\right)\left(a_{o} r\right)^{-1.6}$ is independent of cosmological parameters. The data can be compared to several different sets of cosmological parameters on a single figure by plotting $\left(\langle D\rangle / D_{*}\right)\left(a_{o} r\right)^{-1.6}$ for each data point and comparing this with $\left(a_{o} r\right)^{-1.6}$ curves obtained for different sets of cosmological parameters, as is shown in Figure 1.

The hypothesis is that, for the correct choice of cosmological parameters, $\langle D\rangle / D_{*}=1$, so that the values of $\left(\langle D\rangle / D_{*}\right)\left(a_{o} r\right)^{-1.6}$ for all 20 radio galaxies should follow a curve that, at each $\mathrm{z}$, is parallel to the curve $\left(a_{o} r\right)^{-1.6}$ obtained for that particular choice of cosmological parameters. Figure 1, shows $\left(\langle D\rangle / D_{*}\right)\left(a_{o} r\right)^{-1.6}$ for the the six new points and 14 original points as a function of $\mathrm{z}$ (the six new points are denoted with stars). Also drawn on this figure are curves of $\left(a_{o} r\right)^{-1.6}$ obtained for specified values of cosmological parameters. In this figure, all of the curves pass through Cygnus A, though this is not required when we 
actually solve for best fitting cosmological parameters. It is clear that curves obtained for a low density universe describe the data points quite well, and the curve describing a universe with $\Omega_{m}=1$ does not follow the data points. Note that actual fits are performed with a free normalization that does not require the predicted curves to pass through Cygnus A, the lowest redshift point. Figure 1 is for illustrative purposes and is not part of any fits.

For all 20 sources, the chi-squared for fitting the ratio to a constant is computed for relevant values of $\beta, \Omega_{m}$, and $\Omega_{\Lambda}$. It is found that the best fit value of $\beta$ is $\beta=1.75 \pm 0.25$. This result is insensitive to the choice of $\Omega_{m}$ and $\Omega_{\Lambda}$, and there appears to be no significant covariance between $\beta$ and cosmological parameters (see Figures ₹a,b).

The confidence contours in the $\Omega_{m}-\Omega_{\Lambda}$ plane are shown in Figures 3 and 1 . The probability associated with a given range of $\Omega_{m}$ and $\Omega_{\Lambda}$ independent of $\beta$ is shown in Figure 3 (referred to as two-dimensional confidence intervals). In Figure 4, the projection of a confidence interval onto either axis $\left(\Omega_{m}\right.$ or $\left.\Omega_{\Lambda}\right)$ indicates the probability associated with a given range of that one parameter, independent of all other parameter choices (referred to as one-dimensional confidence intervals). Both figures illustrate how this method and the data are most consistent with a low density universe; $\Omega_{m} \lesssim 0.15$ with $68 \%$ confidence, $\Omega_{m} \lesssim 0.5$ with $90 \%$ confidence, and $\Omega_{m} \lesssim 1.0$ with $99 \%$ confidence. The constraints on $\Omega_{\Lambda}$ are not as strong, and values of $\Omega_{\Lambda}$ from zero to unity are consistent with the data.

The best fit value of $\beta=1.75 \pm 0.25$ is consistent with the previous estimates of $\beta \simeq 1.5 \pm 0.5$ (Daly 1994), and $\beta \simeq 2.1 \pm 0.6$ (Guerra \& Daly 1996, 1998), but with significantly reduced uncertainties. Similarly, the constraints on cosmological parameters are consistent with previous estimates, (Daly 1994; Guerra \& Daly 1996, 1998; Guerra 1997; Daly, Guerra, \& Wan 1998) but with smaller error bars. It is apparent in Figures 3 and 6 that these data and method strongly favor a low density universe; a universe where $\Omega_{m}=1$ is ruled out with $99.0 \%$ confidence independent of $\Omega_{\Lambda}$ and $\beta$. This will be discussed in more detail in 迴.

\subsection{Ambient Gas Densities and Beam Powers}

Daly $(1994,1995)$ showed that the radio properties of a powerful extended radio source could be used to estimate the beam power, $L_{j}$, and density of the gas in the vicinity of the source, $n_{a}$. The method is described in detail by Wellman (1997), Wellman, Daly, \& Wan (1997a), Wan (1998), and Wan, Daly, \& Guerra (1998). Values for the ambient gas density and beam power for the 14 sources in the original sample are described in these papers. 
The basic equations are:

$$
L_{j} \propto a_{L}^{2} B_{10}^{2} v_{L},
$$

and

$$
n_{a} \propto \frac{B_{10}^{2}}{v_{L}^{2}} ;
$$

the normalizations are given in the references listed above. The values obtained for the six new radio galaxies in our sample are listed in Table t. Also listed in Table 6 are all the input parameters used to compute $L_{j}, n_{a}$, and $D_{*}$.

\section{Discussion}

A parent population of 70 powerful extended classical double radio galaxies with redshifts between zero and two was used to define the evolution of the mean or characteristic size of these sources as a function of redshift. An independent estimate of the mean or characteristic size of a given source was possible for a subset of 20 of these radio galaxies for which extensive multiple frequency radio data was available. Requiring that the two measures of the mean source size have the same redshift behavior allows a simultaneous determination of three parameters: the one model parameter $\beta$, and the two cosmological parameters $\Omega_{m}$ and $\Omega_{\Lambda}$ (assuming that the only significant cosmological parameters today are the mean mass density, a cosmological constant, and space curvature).

The method was applied to this data set, and interesting new constraints are presented. It is found that the model parameter is very tightly constrained to be $\beta=1.75 \pm 0.25$ (see Figure 2), consistent with previous estimates, and that this model parameter is independent of cosmological parameters. For a value of $\beta=1.75$, the characteristic source size is $D_{*} \propto\left(B_{L} a_{L}\right)^{-7 / 6} v_{L}{ }^{5 / 12}$, which indicates that it is necessary to have multiple-frequency radio data in order to estimate $D_{*}$, owing to its $v_{L}$ dependence.

The data strongly favor a low density universe; a universe with $\Omega_{m}=1$ is ruled out with $99 \%$ confidence, independent of the value of $\Omega_{\Lambda}$ or $\beta$. Either space curvature or a cosmological constant, or both, are allowed. The main conclusion is that $\Omega_{m}$ is low, but, at this point, the method and data do not allow a discrimination between whether space curvature or a cosmological constant is important at the present epoch.

It is interesting to note that the lowest reduced chi-squared obtained is 0.96 for $\Omega_{m} \simeq-0.25$ and $\Omega_{\Lambda} \simeq 0$. This value is slightly greater and closer to unity than the minimum reduced chi-squared obtained by Guerra \& Daly (1998), which indicates that this sample of 20 sources has a reasonable distribution around any model predictions. This 
convergence to unity with increasing sample size suggests that this method and its statistics are reliable.

The best fit for cosmological parameters in the physically relevant half-plane of $\Omega_{m} \gtrsim 0$ is $\Omega_{m}=0$ and $\Omega_{\Lambda}=0.45$ with a reduced chi-squared of 0.98 (also close to unity). However, Figures 3 and 1 clearly show that our results are still consistent $\Omega_{\Lambda}=0$.

The radio data also allow a determination of the density of the ambient gas in the vicinity of each radio source, and the beam power of each source; the values of these quantities are presented. The sources lie in high-density gaseous environments like those found in low-redshift clusters of galaxies. Typical beam powers for the sources are $\sim 10^{45} \mathrm{erg} \mathrm{s}^{-1}$.

Special thanks go to Rick Perley for his aid in extracting data from the VLA archive. The authors would also like to thank Katherine Blundell, Chris Carilli, Miller Goss, Paddy Leahy, Wil van Breugel, and Dave Wilkinson for helpful discussions. It is a pleasure to acknowledge Ingrid Stairs, Rick Balsano, and other members of the pulsar group who graciously allowed us to use their computer facilities. This work was supported in part by the U.S. National Science Foundation, the Independent College Fund of New Jersey, and a grant from W. M. Wheeler III. 


\section{REFERENCES}

Bennett, A. S. 1962, MmRAS, 68, 163

Daly, R. A. 1994, ApJ, 426, 38

Daly, R. A. 1995, ApJ, 454, 580

Daly, R. A. 1996, in IAU 175: Extragalactic Radio Sources, ed. C. Fanti (Dordrecht: Kluwer), 319

Daly, R. A., Guerra, E. J., \& Wan, L. 1998, in Fundamental Parameters in Cosmology, ed. J. Trân Thanh Vân \& Y. Giraud-Heraud, (Paris: Editions Frontieres), in press

Fanaroff, B. L. \& Riley, J. M. 1974, MNRAS, 167, 31

Guerra, E. J. 1997, Ph.D. thesis, Princeton University

Guerra, E. J. \& Daly, R. A. 1996, in Cygnus A: Study of a Radio Galaxy, ed. C. Carilli \& D. Harris (Cambridge: Cambridge University Press), 252

Guerra, E. J. \& Daly, R.A. 1998, ApJ, 493, 536.

Leahy, J. P., Muxlow, T. W., \& Stephens, P. W. 1989, MNRAS, 239, 401

Liu, R., Pooley, G., \& Riley, J. M. 1992, MNRAS, 257, 545

Myers, S. T. \& Spangler, S. R. 1985, ApJ, 291, 52

Wan, L. 1998, Ph.D. thesis, Princeton University

Wan, L. \& Daly, R. A. 1998a, ApJS, 115, 141

Wan, L. \& Daly, R. A. 1998b, ApJ, 499, 614

Wan, L., Daly, R. A., \& Guerra, E. J. 1998, ApJ, submitted

Wan, L., Daly, R. A., \& Wellman, G. F. 1996, in Energy Transport in Radio Galaxies and Quasars, ed. P. Hardee, A. Bridle, \& J. Zensus, (San Francisco: ASP Conf. Proc.), 305

Wellman, G. F. 1997, Ph.D. thesis, Princeton University

Wellman, G. F \& Daly, R. A. 1996a, in Cygnus A: Study of a Radio Galaxy, ed. C. Carilli \& D. Harris (Cambridge: Cambridge University Press), 215 
Wellman, G. F \& Daly, R. A. 1996b, in Cygnus A: Study of a Radio Galaxy, ed. C. Carilli \& D. Harris (Cambridge: Cambridge University Press), 246

Wellman, G. F., Daly, R. A., \& Wan, L. 1997a, ApJ, 480, 79

Wellman, G. F., Daly, R. A., \& Wan, L. 1997b, ApJ, 480, 96 
Table 1. VLA Archive Data Sets

\begin{tabular}{ccccccc}
\hline \hline Source & Band & Program ID & $\begin{array}{c}\text { Listed } \\
\text { Observer }\end{array}$ & $\begin{array}{c}\text { Frequency } \\
(\mathrm{GHz})\end{array}$ & $\begin{array}{c}\text { Array } \\
\text { Config. }\end{array}$ & Date \\
\hline \multirow{2}{*}{ 3C 244.1 } & L & POOL & Pooley, G. & 1.411 & B & $09 / 17 / 82$ \\
& C & AF213 & Fernini, I. & 4.872 & B & $12 / 23 / 91$ \\
3C 337 & L & AR123 & Rudnick, L. & 1.452 & A & $02 / 21 / 85$ \\
& C & AR123 & Rudnick, L. & 4.885 & B & $06 / 01 / 85$ \\
3C 325 & L & AV153 & van Breugel, W. & 1.465 & A & $12 / 05 / 88$ \\
& C & AF213 & Fernini, I. & 4.885 & B & $12 / 23 / 91$ \\
3C 194 & L & AV164 & van Breugel, W. & 1.465 & A & $05 / 11 / 90$ \\
& C & AV164 & van Breugel, W. & 4.885 & A & $05 / 11 / 90$ \\
3C 324 & L & AR123 & Rudnick, L. & 1.452 & A & $02 / 21 / 85$ \\
& C & AR123 & Rudnick, L. & 4.885 & B & $06 / 01 / 85$ \\
3C 437 & L & AV164 & van Breugel, W. & 1.465 & A & $05 / 11 / 90$ \\
& C & AV164 & van Breugel, W. & 4.885 & B & $04 / 21 / 89$ \\
\hline
\end{tabular}

Table 2. Radio Galaxies with $D_{\star}$ Presented Here

\begin{tabular}{|c|c|c|c|c|c|c|}
\hline \multirow[b]{2}{*}{ Source } & \multirow[b]{2}{*}{$z$} & \multirow[b]{2}{*}{ Bin } & \multirow{2}{*}{$\begin{array}{c}\theta \\
(\operatorname{arcsec})\end{array}$} & \multicolumn{2}{|c|}{$r_{\star}{ }^{a}$} & \multirow{2}{*}{$\begin{array}{c}D_{\star}^{{ }^{\mathrm{a}}} \\
\left(h^{-1} \mathrm{kpc}\right)\end{array}$} \\
\hline & & & & $\left(h^{-1} \mathrm{kpc}\right)$ & $\left(h^{-1} \mathrm{kpc}\right)$ & \\
\hline 3C 244.1 & 0.43 & 2 & 50.8 & $151 \pm 16$ & $135 \pm 16$ & $286 \pm 22$ \\
\hline 3C 337 & 0.63 & 3 & 43.5 & $150 \pm 24$ & $65 \pm 7$ & $214 \pm 25$ \\
\hline $3 \mathrm{C} 325$ & 0.86 & 3 & 15.8 & $164 \pm 52$ & $66 \pm 14$ & $230 \pm 54$ \\
\hline 3C 194 & 1.19 & 4 & 14.2 & $105 \pm 22$ & $92 \pm 15$ & $197 \pm 27$ \\
\hline $3 \mathrm{C} 324$ & 1.21 & 5 & 10.2 & $75 \pm 18$ & $\cdots$ & $149 \pm 35$ \\
\hline $3 \mathrm{C} 437$ & 1.48 & 5 & 36.7 & $55 \pm 6$ & $48 \pm 5$ & $103 \pm 8$ \\
\hline
\end{tabular}

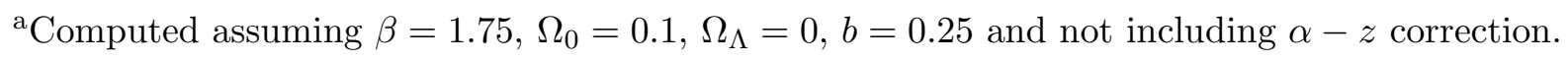

${ }^{\mathrm{b}} r_{\star}$ for only one bridge. 
Table 3. The Average Lobe-Lobe Sizes for Powerful 3CR Radio Galaxies.

\begin{tabular}{cccccc}
\hline \hline & & \multicolumn{3}{c}{$\langle D\rangle\left(h^{-1} \mathrm{kpc}\right)$} \\
\cline { 3 - 6 } Bin & $z$ Range & Sources & $\Omega_{o}=1.0, \Omega_{\Lambda}=0.0$ & $\Omega_{o}=0.1, \Omega_{\Lambda}=0.0$ & $\Omega_{o}=0.1, \Omega_{\Lambda}=0.9$ \\
\hline \multirow{2}{*}{1} & $0.0-0.3$ & 3 & $66 \pm 14$ & $68 \pm 14$ & $72 \pm 13$ \\
2 & $0.3-0.6$ & 13 & $202 \pm 45$ & $224 \pm 50$ & $259 \pm 57$ \\
3 & $0.6-0.9$ & 23 & $148 \pm 17$ & $174 \pm 20$ & $209 \pm 24$ \\
4 & $0.9-1.2$ & 16 & $107 \pm 24$ & $133 \pm 29$ & $165 \pm 36$ \\
5 & $1.2-1.6$ & 9 & $91 \pm 32$ & $122 \pm 43$ & $152 \pm 53$ \\
6 & $1.6-2.0$ & 6 & $66 \pm 19$ & $92 \pm 26$ & $114 \pm 33$ \\
\hline
\end{tabular}


Table 4. Summary of New Source Properties

\begin{tabular}{cccccccc}
\hline \hline Source & $z$ & $a_{L}{ }^{\mathrm{a}}$ & $B_{10}{ }^{\mathrm{b}}$ & $B_{25}{ }^{\mathrm{c}}$ & $v_{L}{ }^{\mathrm{d}}$ & $n_{a}{ }^{\mathrm{e}}$ & $\log L_{j}{ }^{\mathrm{f}}$ \\
\hline 3C 244.1 & 0.43 & $5.5 \pm 0.3$ & $3.5 \pm 0.2$ & $2.5 \pm 0.2$ & $1.1 \pm 0.2$ & $1.0 \pm 0.4$ & $44.35 \pm 0.10$ \\
& & $4.7 \pm 0.4$ & $5.3 \pm 0.3$ & $3.2 \pm 0.2$ & $1.7 \pm 0.3$ & $1.0 \pm 0.4$ & $44.75 \pm 0.11$ \\
3C 337 & 0.63 & $6.2 \pm 0.5$ & $3.5 \pm 0.2$ & $2.3 \pm 0.1$ & $1.5 \pm 0.5$ & $0.6 \pm 0.4$ & $44.57 \pm 0.15$ \\
& & $10.5 \pm 0.4$ & $3.8 \pm 0.2$ & $2.8 \pm 0.2$ & $1.1 \pm 0.2$ & $1.2 \pm 0.4$ & $44.99 \pm 0.10$ \\
3C 325 & 0.86 & $3.3 \pm 1.1$ & $6.8 \pm 0.9$ & $4.0 \pm 0.3$ & $2.0 \pm 0.5$ & $1.2 \pm 0.6$ & $44.73 \pm 0.23$ \\
& & $4.4 \pm 0.8$ & $12.7 \pm 1.0$ & $4.6 \pm 0.3$ & $3.0 \pm 0.8$ & $1.9 \pm 1.0$ & $45.70 \pm 0.17$ \\
3C 194 & 1.19 & $4.5 \pm 1.0$ & $7.1 \pm 0.6$ & $5.0 \pm 0.3$ & $1.8 \pm 0.3$ & $1.6 \pm 0.6$ & $45.00 \pm 0.16$ \\
& & $5.6 \pm 0.8$ & $6.7 \pm 0.5$ & $5.6 \pm 0.4$ & $2.0 \pm 0.3$ & $1.2 \pm 0.4$ & $45.16 \pm 0.13$ \\
3C 324 & 1.21 & $4.5 \pm 0.9$ & $10.3 \pm 0.9$ & $\cdots$ & $2.1 \pm 0.7$ & $2.4 \pm 1.5$ & $45.38 \pm 0.19$ \\
3C 437 & 1.48 & $11.4 \pm 0.8$ & $6.9 \pm 0.4$ & $4.2 \pm 0.2$ & $4.6 \pm 0.8$ & $0.2 \pm 0.1$ & $46.18 \pm 0.10$ \\
& & $10.5 \pm 0.8$ & $9.4 \pm 0.6$ & $6.7 \pm 0.4$ & $6.0 \pm 1.0$ & $0.2 \pm 0.1$ & $46.49 \pm 0.10$ \\
\hline
\end{tabular}

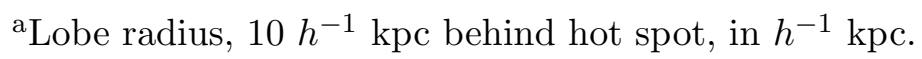

${ }^{\mathrm{b}}$ Minimum energy magnetic field, $10 h^{-1} \mathrm{kpc}$ behind hot spot, in $h^{2 / 7} 10^{-5} \mathrm{G}$.

${ }^{\mathrm{c}}$ Minimum energy magnetic field, $25 h^{-1} \mathrm{kpc}$ behind hot spot, in $h^{2 / 7} 10^{-5} \mathrm{G}$.

${ }^{\mathrm{d}}$ Lobe advance speed, in $10^{-2} c$.

eAmbient gas density, in $10^{-3} h^{1 / 2} \mathrm{~cm}^{-3}$.

${ }^{\mathrm{f}}$ Logarithm of the luminosity in directed kinetic energy, in $h^{-2} \mathrm{erg} \mathrm{s}^{-1}$. 


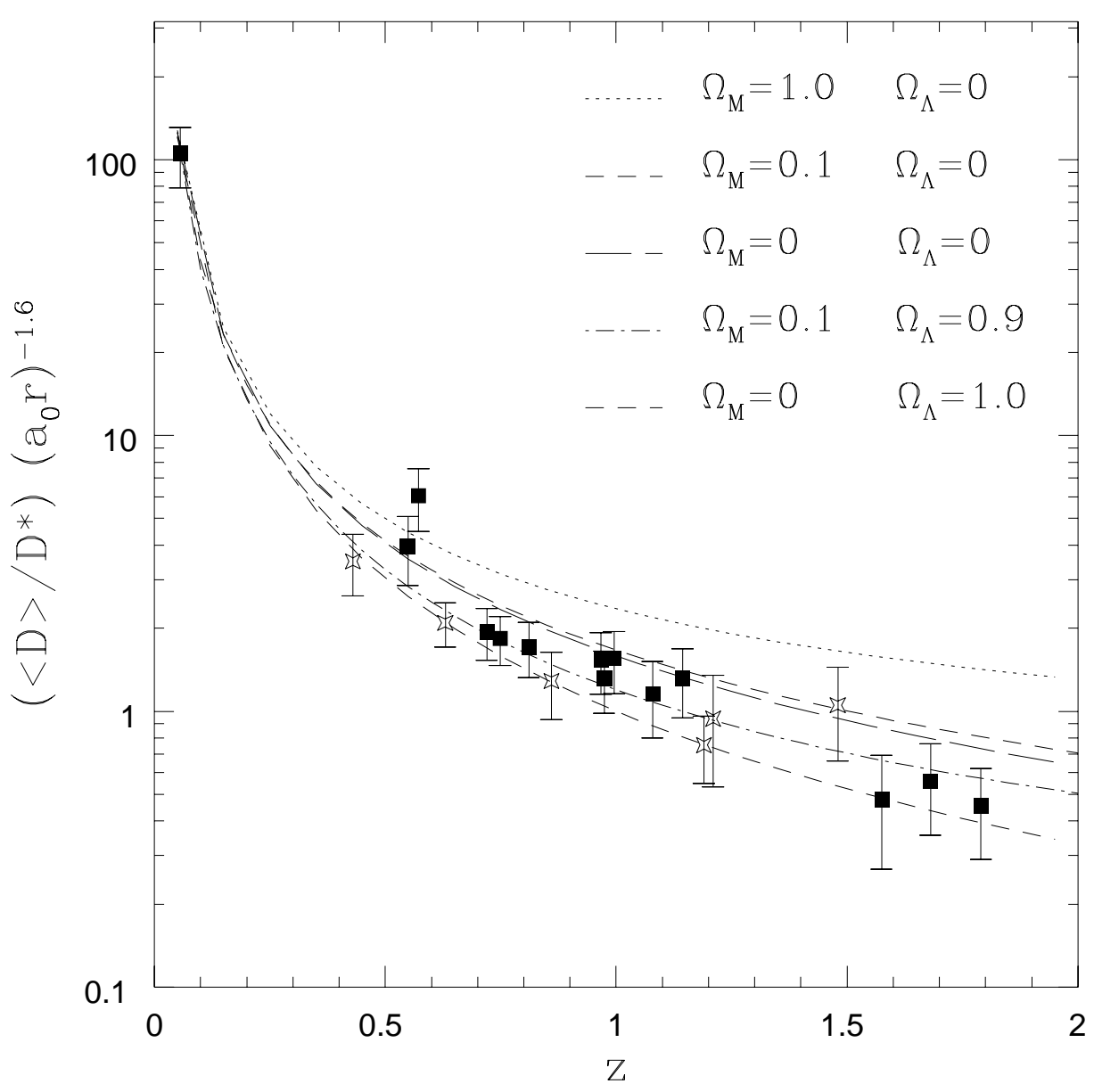

Fig. 1.- The quantity $\left(\langle D\rangle / D_{*}\right)\left(a_{o} r\right)^{-1.6}$, computed assuming $\beta=1.75, b=0.25$ and not including $\alpha-z$ correction. This measured quantity is independent $\Omega_{m}$ and $\Omega_{\Lambda}$. Open Stars denote the six new data points from the VLA archive. For different choices of $\Omega_{m}$ and $\Omega_{\Lambda}$, the predicted redshift evolution of $\left(a_{o} r\right)^{-1.6}$ is plotted for comparison. 


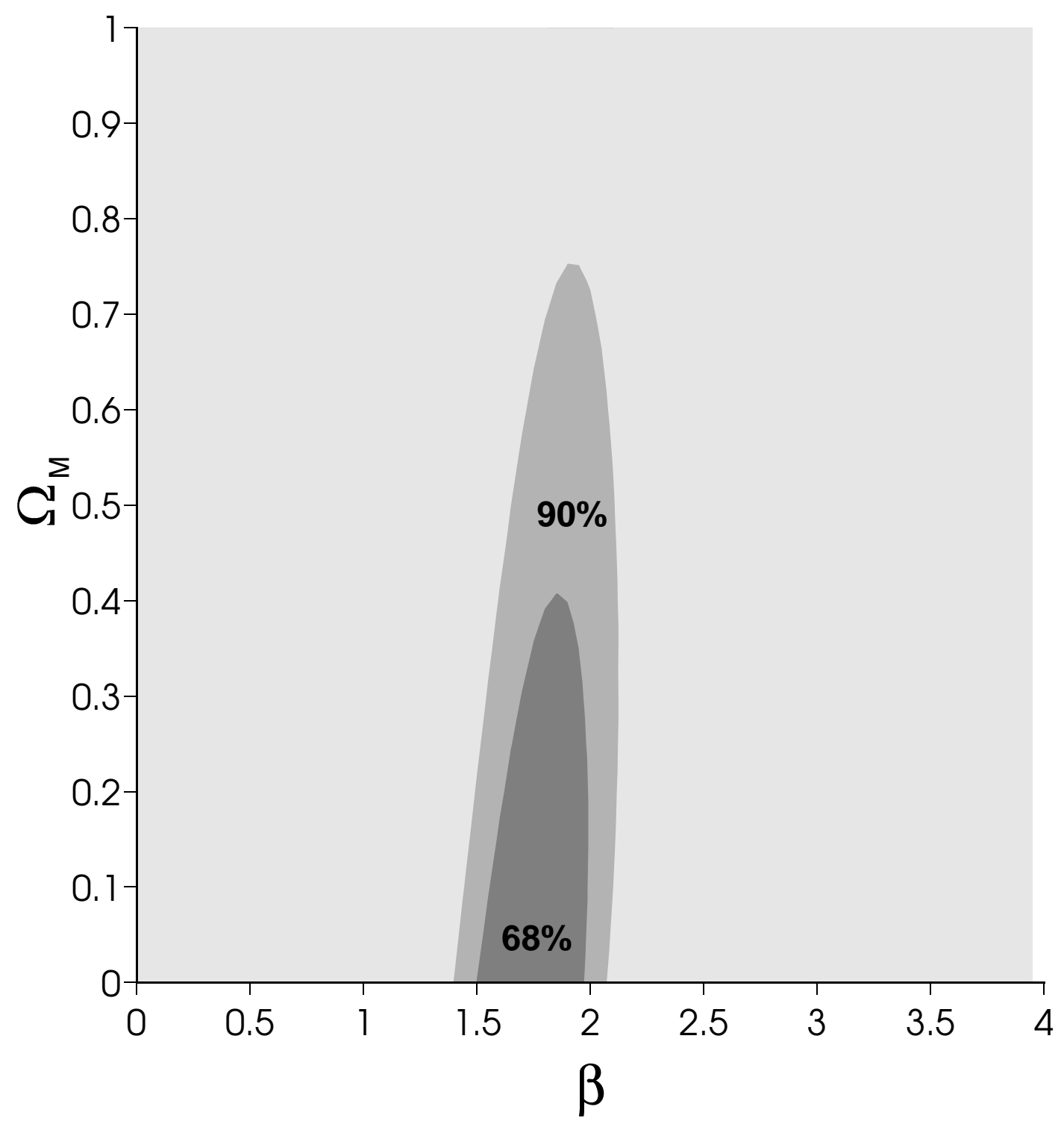

Fig. 2.- The $68 \%$ and $90 \%$ confidence intervals for $\beta$ and $\Omega_{m}$, where (a) $\Omega_{\Lambda}=0$ (no cosmological constant)... 


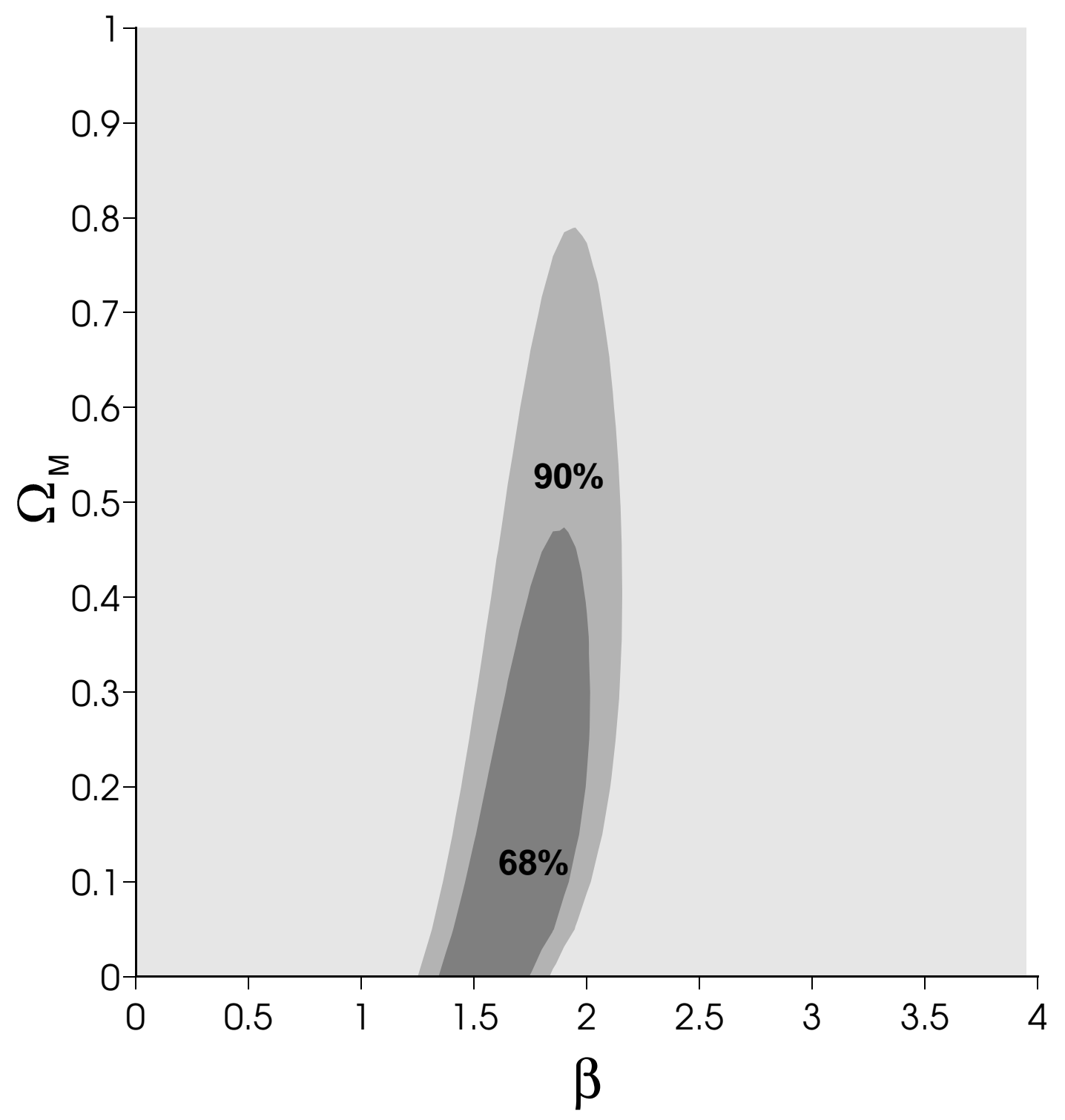

Fig. 2. - ...and (b) $\Omega_{\Lambda}=1-\Omega_{m}$ (spatially flat universe). 


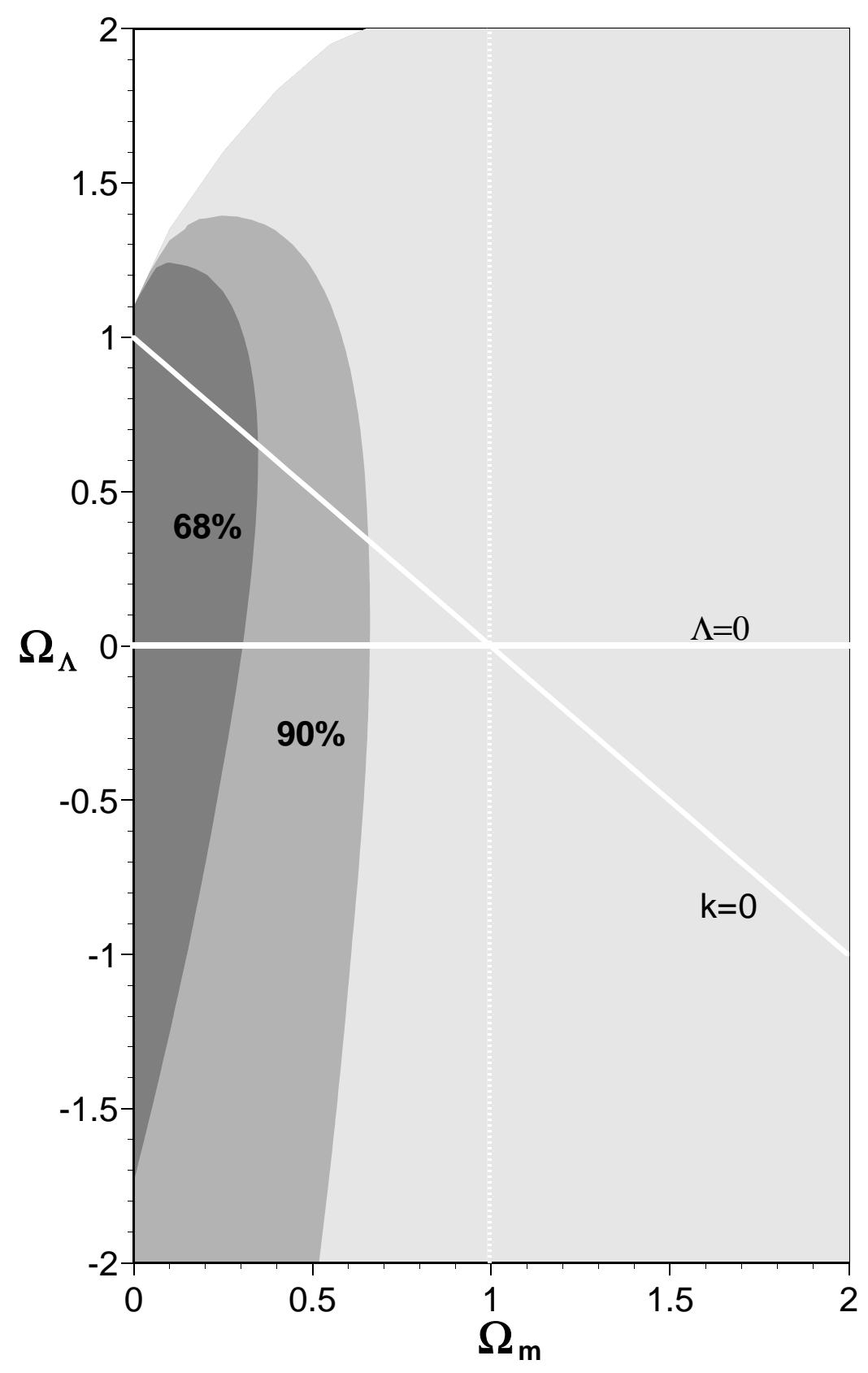

Fig. 3.- The $68 \%$ and $90 \%$ confidence intervals for ranges of both $\Omega_{m}$ and $\Omega_{\Lambda}$, independent of $\beta$. (Two-dimensional) 


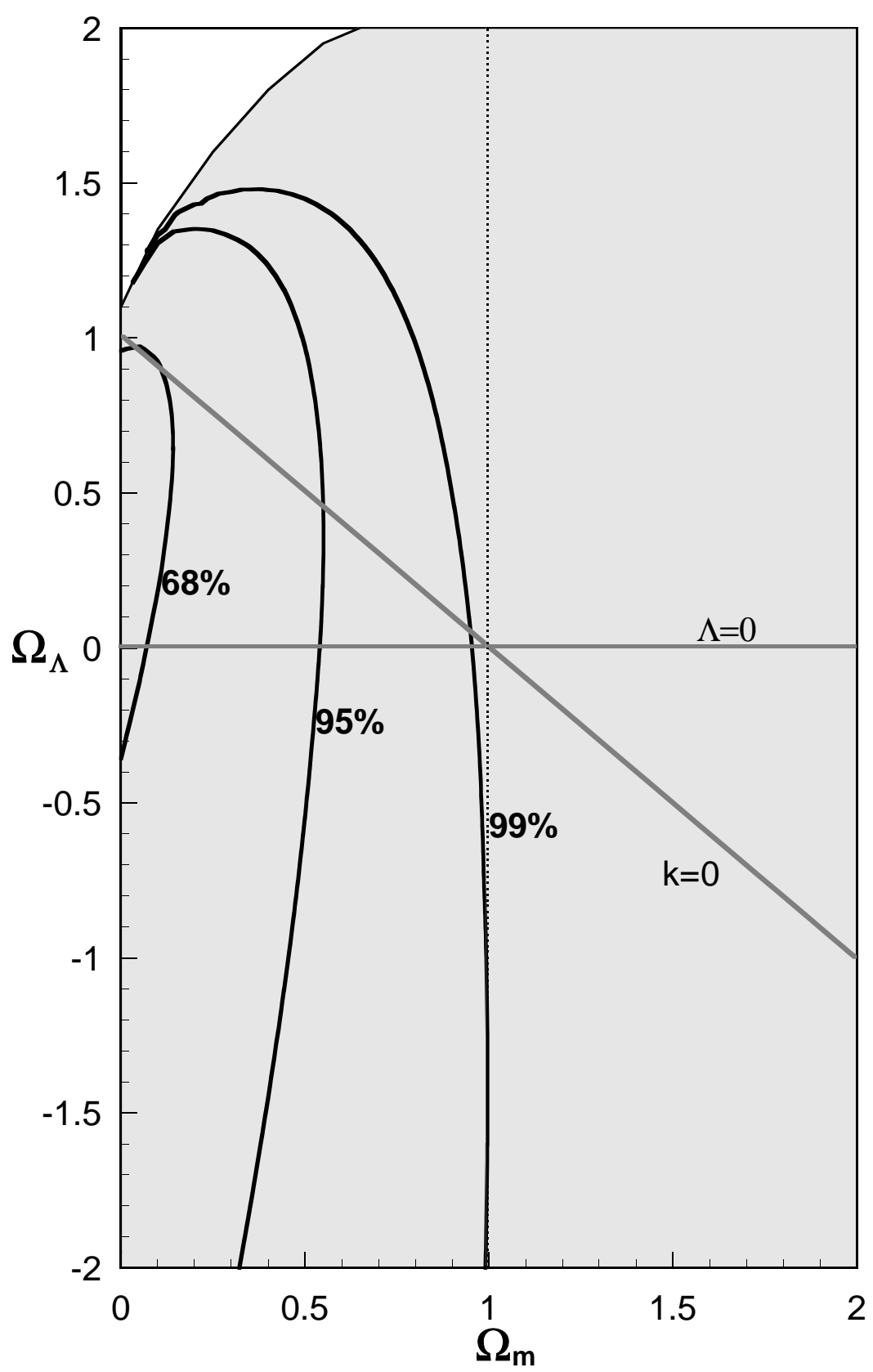

Fig. 4.- The projections of the $68 \%, 95 \%$, and $99 \%$ confidence intervals onto either axis $\left(\Omega_{m}\right.$ or $\left.\Omega_{\Lambda}\right)$ indicates the probability associated with the range in that one parameter, independent of all other parameter choices. (One-dimensional) 